OPEN ACCESS

Edited by:

Niels Janssen

Universidad de La Laguna, Spain

Reviewed by:

Sabrina Aristei,

University of Luxembourg,

Luxembourg

Katharina Spalek,

Humboldt-Universität zu Berlin,

Germany

*Correspondence:

Andrea Krott

a.krott@bham.ac.uk

Specialty section:

This article was submitted to

Language Sciences,

a section of the journal

Frontiers in Psychology

Received: 19 November 2018

Accepted: 12 March 2019

Published: 28 March 2019

Citation:

Krott A, Medaglia MT and

Porcaro C (2019) Early and Late

Effects of Semantic Distractors on Electroencephalographic Responses

During Overt Picture Naming.

Front. Psychol. 10:696.

doi: 10.3389/fpsyg.2019.00696

\section{Early and Late Effects of Semantic Distractors on Electroencephalographic Responses During Overt Picture Naming}

\author{
Andrea Krott' ${ }^{1 \star}$, Maria Teresa Medaglia² and Camillo Porcaro ${ }^{1,2,3,4}$ \\ ${ }^{1}$ Centre for Human Brain Health, School of Psychology, University of Birmingham, Birmingham, United Kingdom, ${ }^{2}$ Institute \\ of Cognitive Sciences and Technologies (ISTC) - National Research Council (CNR), Rome, Italy, ${ }^{3}$ S. Anna Institute and \\ Research in Advanced Neurorehabilitation (RAN), Crotone, Italy, ${ }^{4}$ Department of Information Engineering, Università \\ Politecnica delle Marche, Ancona, Italy
}

This study investigated the nature of the interference effect of semantically related distractors in the picture-word interference paradigm, which has been claimed to be caused by either competition between lexical representations of target and distractor or by a late response exclusion mechanism that removes the distractor from a response buffer. EEG was recorded while participants overtly named pictures accompanied by categorically related versus unrelated written distractor words. In contrast to previous studies, stimuli were presented for only $250 \mathrm{~ms}$ to avoid any re-processing. ERP effects of relatedness were found around 290, 470, 540, and 660 ms post stimulus onset. In addition, related distractors led to an increase in midfrontal theta power, especially from about 440 to $540 \mathrm{~ms}$, as well as to decreased high beta power between 40 and $110 \mathrm{~ms}$ and increased high beta power between 275 and 340 ms post stimulus onset. Response-locked analyses showed no differences in ERPs, however increased low and high beta power for related distractors in various time windows, most importantly a high beta power increase between -175 and -155 ms before speech onset. These results suggest that the semantic distractor effect is a combination of various effects and that the lexical competition account and the response exclusion account each capture a part, but not all aspects of the effect.

Keywords: electroencephalography (EEG), oscillations, word production, picture-word interference paradigm, semantic competition, lexical competition, response exclusion, overt picture naming

\section{INTRODUCTION}

One of the core processes of language production is the selection of the appropriate representations for a particular concept from our mental lexicons. Most models of speech production agree that word production involves a number of processing stages, namely at a conceptual level, a lexical level, a morphophonological level and a phonetic/articulatory level (e.g., Dell, 1986; Levelt et al., 1999). Models also generally agree that during the lexical access of a target word (e.g., banana) not only its lexical representation is activated, but that of semantically related words (e.g., apple, orange, pear etc.) (Dell, 1986; Caramazza, 1997; Levelt et al., 1999; Goldrick and Rapp, 2002; Indefrey, 2011). However, models do disagree on whether the co-activated lexical representations compete 
for selection or not. Some models do assume lexical selection by competition (e.g., Schriefers et al., 1990; Roelofs, 1992; Starreveld and La Heij, 1996; Levelt et al., 1999; Bloem and La Heij, 2003; Abdel Rahman and Melinger, 2009). More precisely, they assume that the co-activation of semantically related representations interferes with the production of the target word, slowing down the production process and leading to slower word production. This is especially the case if a competitor is highly activated. The higher the activation of the competitor, the slower the production of the target. The exact mechanism causing the slowing varies between models. For instance, Cutting and Ferreira (1999) proposed the existence of lateral inhibitory links between lexical representations, and the WEAVER++ model (Roelofs, 1992) proposes that a word is selected for production only once its activation exceeds those of other representations by a critical amount. In contrast, other models (e.g., Dell, 1986; Caramazza, 1997) assume that the co-activation of other lexical items does not affect the selection of the target word. Instead, only the target's own activation level affects the speed of its naming process. However, a co-activated word might be selected for production if its activation is higher than that of the target. This would lead to a speech error.

The assumption of lexical selection by competition was originally supported by findings of a particular experimental paradigm, namely the picture-word interference (PWI) paradigm. In this paradigm, participants are asked to name pictures with visually or auditorily superimposed distractor words. Numerous studies have found that distractors from the same semantic category as the target (e.g., 'cat' as a distractor to the target 'fish') slow down naming times when compared to unrelated distractors (e.g., 'cup') (e.g., Lupker, 1979; Glaser and Düngelhoff, 1984; Schriefers et al., 1990). This finding has been called the "semantic interference effect." The lexical selection by competition interpretation of the semantic interference effect states that the representation of an unrelated distractor receives activation from the distractor word only, while a semantically related distractor receives activation from both the distractor word and the target due to spreading activation at the conceptual level. This is assumed to result in higher activation of related than unrelated distractors and as a consequence to stronger competition with the target. This in turn slows down response times. Importantly, the competition between target and related words is assumed to take place at lexical level.

This traditional lexical competition account of the semantic interference effect has been questioned. The alternative account that has been proposed is the so-called "response exclusion account." This account extends models that do not assume lexical competition (e.g., Caramazza, 1997). It proposes that a semantic distractor affects the naming of a picture at two stages: during early semantic processing of the picture and a late processing stage, i.e., when a fully planned response to the distractor needs to be removed from a response buffer (Finkbeiner and Caramazza, 2006; Mahon et al., 2007; Janssen et al., 2008; Dhooge and Hartsuiker, 2010, 2012). The early semantic effect is a semantic priming effect and thus facilitatory. The semantic interference effect and thus the slowing down is caused by the need to remove the distractor from the response buffer. This buffer is occupied by the response to the written distractor because it is processed faster than the picture. The removal is assumed to be affected by general semantic constraints or "response-relevant criteria." It is checked whether the word in the buffer meets these constraints in order to be an acceptable response. Because unrelated distractors are less response relevant, their removal is easier and faster than the removal of a related distractor. This leads to slower responses for related than unrelated distractors.

Evidence for the accounts has primarily been found by manipulating the type of the distractor of the PWI paradigm (e.g., Alario et al., 2000; Miozzo and Caramazza, 2003; Abdel Rahman and Melinger, 2007; Dhooge and Hartsuiker, 2010; Roelofs et al., 2011). But both lexical competition accounts and the response exclusion account are able to explain the behavioral findings that have been reported to date (Roelofs et al., 2011). Deeper insights might therefore come from neuroimaging studies or studies using electroencephalography (EEG).

Studies using functional magnetic resonance imaging (fMRI) have suggested that the two accounts are not necessarily mutual exclusive explanations of the semantic interference effect (de Zubicaray et al., 2012a,b). For instance, de Zubicaray et al. (2012b) manipulated frequency and age-of-acquisition of distractors in a PWI paradigm. The age-of-acquisition effect was associated with the left middle and posterior middle temporal gyrus (MTG), areas generally thought to play a role in lexical semantic processing (e.g., Indefrey and Levelt, 2004; Acheson et al., 2011; Indefrey, 2011). This finding is therefore in accordance with the assumption of the lexical competition account that distractors compete with the target at a lexical level. In contrast, manipulating distractor frequency affected neural activity in posterior superior temporal cortices (pSTG) and left premotor cortices. The pSTG has been related to the retrieval of phonological word forms / phonological encoding, and premotor cortical areas have been related to articulation (e.g., Indefrey and Levelt, 2004; Wilson et al., 2009; Acheson et al., 2011; Indefrey, 2011). Because these processes are assumed to occur close to response, the involvement of these areas is in accordance with the assumption of the response exclusion account of a late removal of a fully planned distractor from a response buffer.

Because the two opposing accounts of the PWI effect make different predictions concerning the timing of the effect, EEG with its strong temporal resolution seems to be particularly suited to distinguish between the accounts. While the lexical competition account predicts an effect during the time of lexical selection processes, the response exclusion account predicts a late effect close to speech onset due to the exclusion of the distractor from the output buffer. A number of studies have studied ERPs and oscillatory EEG activity to trace the time course of the PWI effect (e.g., Greenham and Stelmack, 2001; Hirschfeld et al., 2008; Dell'Acqua et al., 2010; Aristei et al., 2011; Hoshino and Thierry, 2011; Blackford et al., 2012; Piai et al., 2012; Zhu et al., 2015; Shitova et al., 2016, 2017; Wong et al., 2017; Rose et al., 2018). The dominant ERP pattern of results seems to suggest a modulation of the N400 component (Dell'Acqua et al., 2010; Blackford et al., 2012; Wong et al., 2017; Zhu et al., 2015). Unrelated distractors (e.g., picture of a banana with the superimposed distractor 'house') compared to related category 
distractors (distractor 'apple') have been found to show a more negative N400 component roughly between 250 and $450 \mathrm{~ms}$, an N400-like effect (see also Shitova et al., 2016, 2017 for identity distractors, e.g., distractor 'banana'). Given the timing of these effects, they have been interpreted as being related to lexicalsemantic access and therefore in line with the lexical selection by competition hypothesis (but see Blackford et al., 2012).

While a N400 modulation is a common result, ERP differences between semantically related and unrelated distractors have not always been found (Greenham and Stelmack, 2001; Hirschfeld et al., 2008; Aristei et al., 2011; Piai et al., 2012; Shitova et al., 2016) and the nature of the ERP effects that have been reported varies (Dell'Acqua et al., 2010; Aristei et al., 2011; Hoshino and Thierry, 2011; Zhu et al., 2015; Wong et al., 2017; Rose et al., 2018). Hoshino and Thierry (2011) reported a much shorter effect at 350-400 ms, in addition to an earlier effect during 200-260 ms (reduced negative deflection for related distractors compared to unrelated distractors). However, their participants were SpanishEnglish bilinguals, meaning that their effects might partly reflect interference and/or inhibition of their other language. Dell'Acqua et al. (2010) reported an additional negativity between 50 and $200 \mathrm{~ms}$, which they interpreted as being due to feedback processes from the semantics of the picture to the processing of the distractor word. While the studies presented so far all found reduced negative deflections for semantically related compared to unrelated distractors, Aristei et al. (2011) found the opposite effect between 200 and $400 \mathrm{~ms}$. But their paradigm was more complex, as they presented stimuli in semantically related and unrelated blocks. This is also the only study that found effects when presenting distractors auditorily instead of visually. Rose et al. (2018) reported an anterior negativity for related distractors roughly between 340 and $450 \mathrm{~ms}$. Furthermore, studies varied with respect to the distribution of the effects. While some found wide-spread central(-parietal) effects that resemble N400 effects (Dell'Acqua et al., 2010; Hoshino and Thierry, 2011; Zhu et al., 2015; Rose et al., 2018), others found (additional) anterior effects (Aristei et al., 2011; Blackford et al., 2012; Wong et al., 2017; Rose et al., 2018).

Leaving the differences between the findings of previous ERP studies using the PWI paradigm aside, in almost all studies, stimuli (i.e., pictures and distractors) were visually presented and remained on the screen until participants responded (but see Aristei et al., 2011; Blackford et al., 2012). This opens up the possibility that pictures and words are processed again, for instance, during later monitoring processes. This in turn means that ERP effects might partly be affected by such re-processing. We decided to avoid any effects of re-processing by adapting a variant of the paradigm where the stimuli are presented on the screen for a much shorter time (e.g., Schriefers et al., 1990; Zwitserlood et al., 2000), namely for $250 \mathrm{~ms}$ only. This also means that any potential late effects that we would find cannot be due to the re-processing of the stimuli.

Furthermore, almost all ERP effects for related versus unrelated distractors reported to date ended before 400/450 ms (550 ms in case of Blackford et al., 2012) and are, at least in terms of timing, in line with the "selection by competition" hypothesis [but see Blackford et al.'s (2012) interpretation]. In contrast to
ERP findings so far, the response exclusion account predicts an effect close to speech onset. To our knowledge, only one PWI study to date has investigated such late ERP responses (Wong et al., 2017), most likely due to a fear of "distortion" of the EEG during this time window by speech motor artifacts (but see Piai et al., 2014, for a response-locked time-frequency analysis in a related paradigm). Wong et al. (2017) investigated late effects by a response-locked analysis. They found an ERP effect only when comparing associative distractors (semantically but not categorically related to the picture) with unrelated distractors, not for categorical distractors, as of interest here. Since the response exclusion account makes predictions about processes very close to responses, it is important to study these later processes with the right approach, i.e., ideally with a response-locked analysis.

There are relatively few studies that have investigated oscillatory EEG activity during a PWI study (Piai et al., 2012; Shitova et al., 2017). Piai et al. (2012) reported a central power increase in the beta band $(12-30 \mathrm{~Hz})$ for categorically related relative to unrelated distractors between 230 and $370 \mathrm{~ms}$. Shitova et al. (2017) reported a midfrontal theta power increase (4$6 \mathrm{~Hz}$ ) for semantically related versus identical distractors from about $200 \mathrm{~ms}$ post stimulus onset until end of the trial (see also theta activity increase in the MEG study by Piai et al., 2014). The beta activity increase for categorically related versus unrelated distractors in Piai et al. (2012) study fits the selection by competition assumption due to its timing. The long-lasting theta power increase from $\sim 200 \mathrm{~ms}$ by Shitova et al. (2017), on the other hand, suggests ongoing heightened cognitive control from about 200 ms onward (e.g., Cavanagh and Frank, 2014) and might therefore mean that neither the lexical selection account nor the response exclusion account capture all aspects of the PWI effect. It seems that a 'conflict' between the picture and the distractor is identified early during speech preparation, leading to this heightened control. The maintained increased control right up to speech onset suggests that enhanced cognitive control might be required during various stages of speech preparation, including lexical selection, but also during later processing stages. Taken together, investigating oscillatory EEG activity can provide additional information about the timing of processes in the PWI paradigm and therefore potential evidence for either or both of the opposing accounts of the PWI effect.

In sum, since leaving stimuli in the PWI paradigm on the screen until response could lead to re-processing, we presented stimuli for only $250 \mathrm{~ms}$. In addition, previous EEG studies have almost exclusively studied ERPs locked to the stimulus and have focused on 'early' effects. While this approach can provide evidence for the lexical competition account, it is limited in its ability to reveal evidence for the response exclusion account, which is expected to occur close to speech onset due to the exclusion of the distractor from the output buffer. In order to minimize any contamination of speech motor artifacts, we used a muscle artifact attenuation procedure, namely Speech Artifact Removal by Independent Component Analysis (SARICA) (Porcaro et al., 2015). Furthermore, we investigated effects for both ERPs and oscillatory activity in order to widen the search space. In line with the predictions of the two accounts of the PWI effect, we expected to see effects during the time window of lexical 
processing and/or after the preparation of a phonological word, thus close to speech onset.

\section{MATERIALS AND METHODS}

\section{Participants}

Eighteen participants (mean age 23.3, SD 3.7, 10 males) took part in the experiment and received either course credit or $£ 20$ for their participation. All were right-handed determined by the Edinburgh handedness inventory (Oldfield, 1971). All had normal or corrected-to-normal vision and were monolingual native English speakers. Ethical approval for the research was obtained from the Ethics Board of the School of Psychology at Birmingham University.

\section{Materials}

Twenty-four line-drawings of common objects were selected from the Snodgrass and Vanderwart (1980) picture set. They were paired with semantically related distractor words taken from the same semantic category as the target [e.g., banana (target picture) - orange (distractor word)]. For category membership, we followed Abdel Rahman and Melinger (2007) definition as items that share a semantic category node (e.g., fruit) and specific features (e.g., grows on trees, is sweet etc). We chose from a wide range of categories. Unrelated target-distractor pairs were created by re-pairing targets and distractor words (see Appendix A for a complete list of material). This ensured that related and unrelated distractors as a group were perfectly matched for all possible variables (length, frequency etc.).

\section{Procedure}

Participants were seated in a quiet and normally illuminated test room $1 \mathrm{~m}$ away from a $17^{\prime \prime}$ monitor with a resolution of $800 \times 600$ pixels. They first familiarized themselves with the pictures and their names by studying a picture booklet. In the experiment, participants saw the pictures with superimposed written distractor words as shown in Figure 1. They were instructed to name the pictures, while ignoring the distractor words, and to respond as fast and as accurately as possible. E-Prime (Psychological Software Tools, Inc.) was used to control stimulus presentation and data collection. Responses were recorded for off-line error analysis and response times were measured using a voicekey (PST SRBox). Each trial began with the presentation of a fixation cross for $800 \mathrm{~ms}$, followed by a stimulus appearing for $250 \mathrm{~ms}$ and a subsequent empty screen. This prevented participants from re-reading the distractor word during later processing stages (e.g., during monitoring) and also minimized eye movements. The maximum response time was $2200 \mathrm{~ms}$ and each trial lasted up to $3000 \mathrm{~ms}$. Participants were instructed to keep movements other than speaking to a minimum and to try to blink only after providing a response.

Two stimulus lists were created, each featuring all objects, combined with one of the distractors. Half of the objects in each list were combined with the related distractor, and the other half with the unrelated distractor. The order of the items within the lists was random. In order to increase the number of trials for the analysis, each participant saw each list eight times in alternation, leading to 192 trials per condition (total 384 trails). The order of the lists was counterbalanced across participants. There was a short pause after the completion of every second list. The experiment lasted for about $75 \mathrm{~min}$ (including breaks).

\section{EEG and EMG Recording Parameters}

Electroencephalograms (EEG) were acquired using a 128 channel BioSemi Active Two EEG system, with electrodes placed in a nylon cap according to the 10-5 system (Oostenveld and Praamstra, 2001). Horizontal and vertical electrooculograms and upper and lower lip electromyograms (EMG) were monitored by bipolar derivations. The data were sampled at $512 \mathrm{~Hz}$. They were off-line referenced to an average of the left and right mastoids, baseline-corrected using the average EEG activity in the interval between $-100 \mathrm{~ms}$ and stimulus presentation and filtered with a band-pass of $0.1-30 \mathrm{~Hz}$ using a finite impulse response (FIR) filter.

\section{DATA ANALYSIS}

For statistical analyses of both the behavioral and ERP data we excluded all trials with missed or incorrect responses, with disfluencies and self-repairs. We also considered responses with reaction times below $250 \mathrm{~ms}$ as voicekey errors and responses above $1800 \mathrm{~ms}$ as outliers and removed those from the analyses $(<0.5 \%$ of the data). For the behavioral data, we compared both speech onset times and number of errors (missed and incorrect responses, disfluencies and self-repairs) for pictures with related and unrelated distractors.

\section{SAR-ICA Analysis}

The data were cleaned from motor artifacts using the previously developed Speech Artifact Removal by Independent Component Analysis (SAR-ICA) procedure (Porcaro et al., 2015), a ICA procedure similar to that used for the attenuation of other artifacts (Barbati et al., 2004; Makeig et al., 2004, Medaglia et al., 2009; Porcaro et al., 2006, 2009, 2011). The SAR-ICA procedure decomposes the signal into independent components (ICs) on the basis of statistical properties of the signal. Using information from averaged trials, single trials, topographical distributions, localizations, and correlations with the lip EMG, ICs are classified into the following clusters: cleaned data, articulatory speech artifacts, environmental noise, and ocular artifacts. The data at scalp electrodes for the cleaned data cluster are obtained by retro-projecting the selected independent components. We have presented the SAR-ICA for the present dataset in detail in Porcaro et al. (2015), together with a validation of our method and a comparison with other methods for motor artifact removal previously reported in the literature. We found a high validity and superiority of our method over previous other approaches. Therefore, cleaning the data from speech motor artifacts using the SAR-ICA method should provide us with overall reliable data, i.e., for both early time-windows and those close to speech onset. 


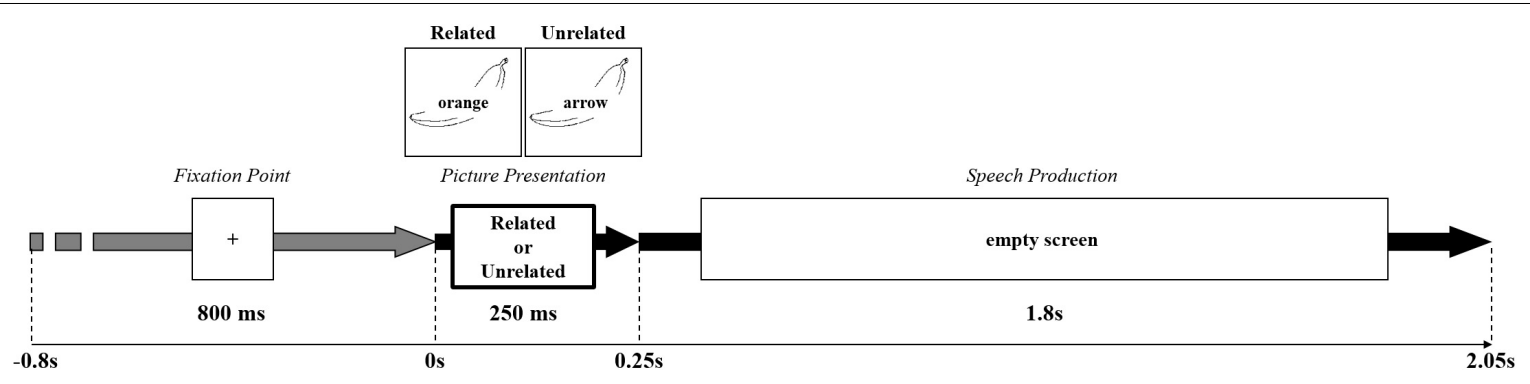

FIGURE 1 | Experimental Procedure. The beginning of the trial was signaled by a "+" appearing in the center of the screen for 800 ms, followed by the picture presentation with a related or unrelated distractor for $250 \mathrm{~ms}$. During speech production, an empty screen appeared for minimum $1800 \mathrm{~ms}$. The Figure shows an example of a semantically related and unrelated distractor.

\section{ICA on ERP Data}

In order to rule out that we are dealing with latency effects instead of amplitude differences for the ERP peaks between 440 and $670 \mathrm{~ms}$, we conducted ICA analyses on the ERPs of related and unrelated distractor conditions.

\section{Cluster-Based Permutation Tests}

To identify effects of distractor relatedness on brain responses, we examined ERP responses to related versus unrelated distractors with both a stimulus-locked and a response-locked analysis. However, we only found significant differences between conditions in the stimulus-locked analysis. We therefore report only those. For the stimulus-locked analysis, we investigated a window that roughly covered processes before speech onset, i.e., 0-800 ms. We split the time window into two equalsized windows, 0-399 ms for early effects and 400-800 ms for late effects. Because ERP differences that had previously been reported for semantic distractors in PWI studies occurred in various time-windows and because ERP differences between categorically related and unrelated distractors closer to responses had previously either not been studied or not been found, we investigated ERP effects by taking an explorative approach. For that we submitted ERPs to repeated measures, two-tailed cluster mass permutation tests with a family-wise alpha level of 0.01 , using the Mass Univariate ERP Toolbox ${ }^{1}$ (Bullmore et al., 1999; Groppe et al., 2011). We included all time points between 0 and $399 \mathrm{~ms}$ and 400 and $800 \mathrm{~ms}$ at all 128 scalp electrodes in the test and used $5.44 \mathrm{~cm}$ (default setting of the toolbox) to determine electrodes as spatial neighbors. Repeated measures $t$-tests were performed for each comparison using the original data and 2500 random within-participant permutations of the data. For each permutation, all $\mathrm{t}$-scores corresponding to uncorrected $p$-values of 0.01 or less were formed into clusters. The sum of the t-scores in each cluster is the "mass" of that cluster and the most extreme cluster mass in each of the 2501 sets of tests was recorded and used to estimate the distribution of the null hypothesis.

\section{Time Frequency Analysis}

Stimulus and response-locked time-frequency event related synchronization and desynchronization (ERS/ERD) analyses

${ }^{1}$ https://openwetware.org/wiki/Mass_Univariate_ERP_Toolbox were performed using a Morlet wavelet, with a constant parameter equal to seven which offered the best compromise between time and frequency resolution, for both related and unrelated conditions and for each subject. Statistical significance of power changes compared to a $500 \mathrm{~ms}$ pre-stimulus baseline for stimulus-locked and from -1500 to $-1000 \mathrm{~ms}$ respect to the speech onset baseline for the response-locked were evaluated with a resampling bootstrap technique and a threshold of $p=0.05$. Non-significant changes were set to zero (Barbati et al., 2008; Porcaro et al., 2017). Given previous findings of theta and beta band changes at mid-frontal areas, especially those for very similar paradigms as the present one (Piai et al., 2012; Shitova et al., 2017), we investigated such time frequency changes at $\mathrm{FCz}$, integrating the time frequency analysis as described above in theta $(4-8 \mathrm{~Hz})$, low beta $(14-21 \mathrm{~Hz})$ and high beta band $(22-30 \mathrm{~Hz})$. Once the theta, low and high beta band dynamics for each subject were obtained for both related and unrelated conditions, a pointwise statistical analysis was performed on the three waveforms conducting two-sample permutation $t$-tests (10,000 permutations) (Porcaro et al., 2019).

\section{RESULTS}

Effects of distractor relatedness on speech onset times were in line with previous findings. Average speech onset times were faster for unrelated (844 ms, SD 206) than related distractors ( $865 \mathrm{~ms}$, SD $217)[t(17)=4.3, p<0.001]$. The average error rates were small and did not significantly differ between related and unrelated distractors (semantically related: $1.4 \%$; unrelated $1.1 \%$ ).

In order to rule out that the repetition of the stimuli had an effect on response times, as found for instance by La Heij and Van den Hof (1995), we conducted an ANOVA with Relatedness (related, unrelated) and Block (Block 1 to Block 8) as independent variables and RTs as dependent variables. While we still found an effect of Relatedness, we did not find a significant effect of Block or a significant Block x Relatedness interaction (both ps $>0.05$ ). Thus, repeating stimuli did not affect the relatedness effect in the behavioural results in our experiment.

Figure 2 shows the results of the cluster-based permutation test of ERP differences on all 128 electrodes between the related and unrelated distractor conditions, and Figure 3 shows averaged 


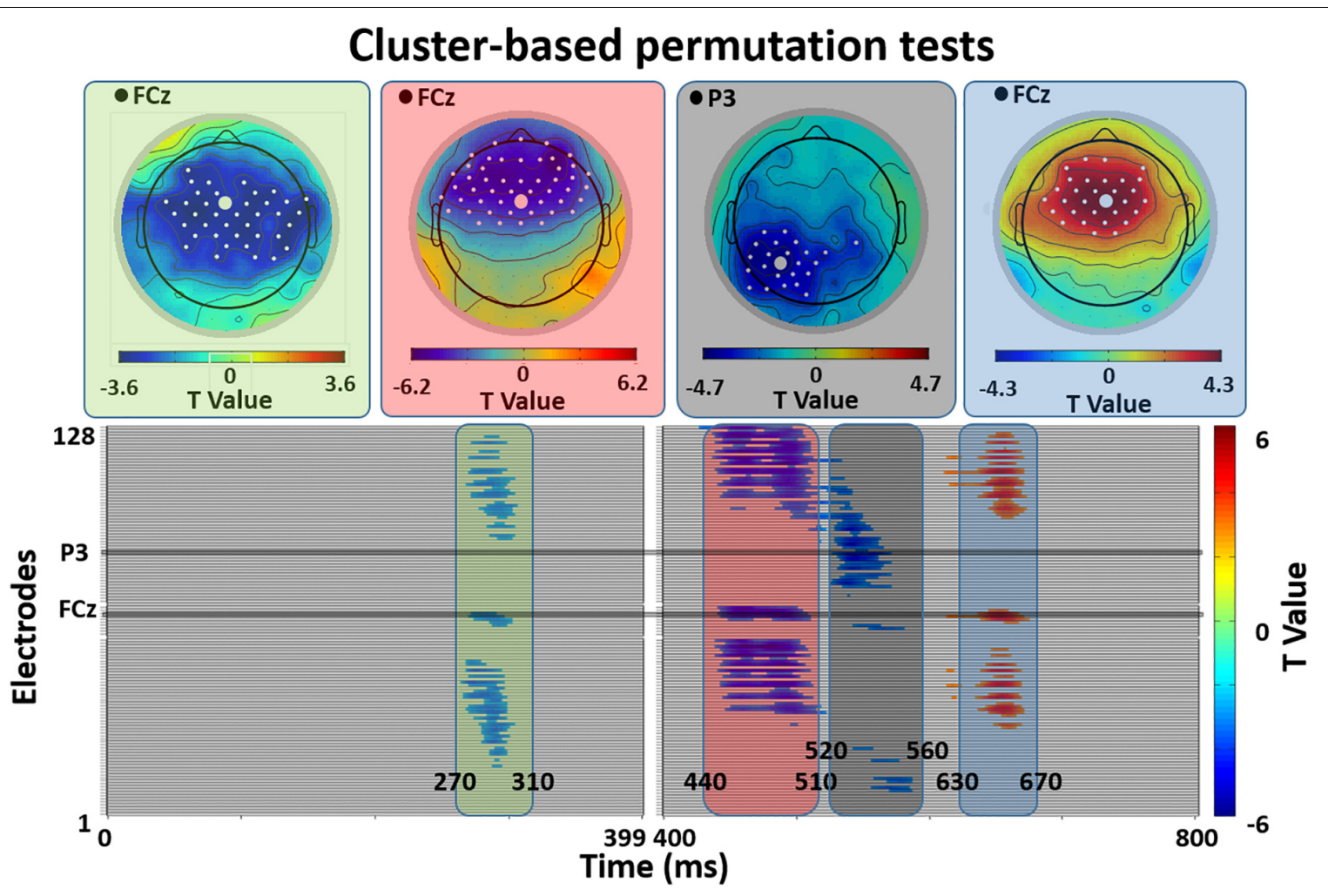

FIGURE 2 | Cluster-based permutation tests of ERP differences between Semantically Related and Unrelated distractor conditions. The lower panels plot significance of ERP differences for all 128 electrodes in four different time-windows post stimulus onset (270-310 ms, 440-510 ms, 520-560 ms, 630-670 ms). The upper panels show the scalp distribution of these effects (related vs. unrelated distractors). White dots indicate electrodes with significant differences. Large white dots indicate the illustrative electrodes in Figure 3.

waveforms for electrodes $\mathrm{FCz}$ and P3 illustrating all effects. We found prominent ERP differences around four peaks: $290 \mathrm{~ms}$ (270-310 ms), $470 \mathrm{~ms}$ (440-510 ms), $540 \mathrm{~ms}$ (520-560 ms), and $660 \mathrm{~ms}(630-670 \mathrm{~ms})$. The window around the $290 \mathrm{~ms}$ peak showed a larger negativity for related than unrelated distractors, widely distributed over the electrode space (see Figure 2, first topography, little white dots represent significant electrodes and big white dots represent the topographic position of the $\mathrm{FCz}$ electrode). For the $470 \mathrm{~ms}$ peak window we found a reduced positivity for related compared to unrelated distractors at anterior electrode sites (see Figure 2, second topography). The direction of the effect was the same at the $540 \mathrm{~ms}$ peak, but located at posterior left sides (see Figure 2, third topography). The $660 \mathrm{~ms}$ peak window showed a reduced negativity for related than unrelated distractors at anterior-central sites (see Figure 2, fourth topography) $)^{2}$.

Similar to the behavioral analysis, we also investigated whether the repetition of stimuli had an effect on the ERP effects. Since a single block only consisted of 24 trials per condition, which is too few for reliable significant condition differences, we conducted the same cluster-based permutation tests as for the total data

\footnotetext{
${ }^{2}$ The same analysis applied before cleaning the EEG from speech motor artifacts (Raw Data only, corrected for eye movements) led to similar results around 290 and $660 \mathrm{~ms}$, but did not lead to any significant differences at 470 and $540 \mathrm{~ms}$.
}

on the first and final (i.e., fourth) quartile of the data. For both subsets of trials, we found very similar effects and in the same time windows as for all trials combined. In addition, there were no significant ERP differences between the first and fourth quartile, for neither the related nor the unrelated condition (see Supplementary Figure S1). Furthermore, we checked whether the later three ERP effects (those between $440 \mathrm{~ms}$ and $670 \mathrm{~ms}$ ) might be due to differences in latencies instead of amplitudes. However, we found that latencies of these effects were not different between related and unrelated distractor conditions [peak around $470 \mathrm{~ms}: t(17)=1.06, p=0.302$; peak around 540 ms: $t(17)=1.04, p=0.314$; peak around $660 \mathrm{~ms}$ : $t(17)=0.39, p=0.701]$. We are therefore indeed dealing with amplitude differences. Furthermore, the waveforms in Figure 3 suggest that related and unrelated conditions might have led to different ERP components. However, this was not the case. Performing ICA analyses on the ERPs of the related and unrelated distractor conditions showed three different components that created the three late peaks for both related and unrelated distractor conditions. This means that the three late peaks were independent from each other (see Supplementary Figure S2), meaning we have no evidence for different underlying mechanisms in the two conditions.

Figure 4 shows the results of the time-frequency analysis at FCz. The stimulus-locked analysis showed increased theta power 


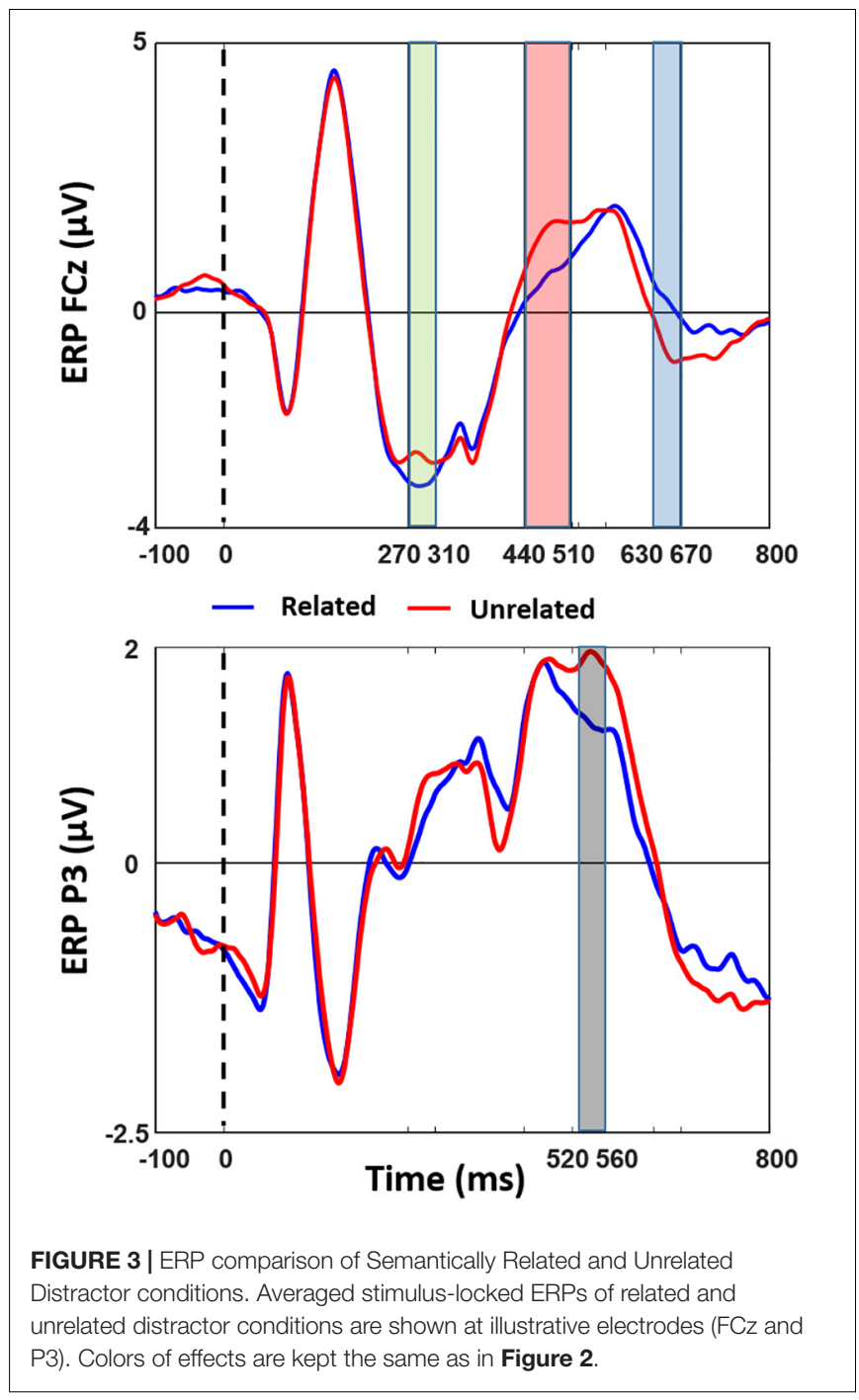

for related versus unrelated distractors from 440 to $540 \mathrm{~ms}$ post stimulus. We also found a decrease in high beta band power for related versus unrelated distractors between 40 and $110 \mathrm{~ms}$ and an increase in high beta band power from 275 to $340 \mathrm{~ms}$ post stimulus onset. The response-locked analysis at $\mathrm{FCz}$ showed various increases in low and high beta band power in the final $\sim 550 \mathrm{~ms}$ before speech onset, with the latest increase from -175 to $-155 \mathrm{~ms}$ before speech onset (other effects: low beta: -545 to $-490 \mathrm{~ms}$ and -290 to $-270 \mathrm{~ms}$ pre-speech onset; high beta: -275 to $-340 \mathrm{~ms}$ post stimulus onset; -365 to $-335 \mathrm{~ms}$ pre speech onset). There were no differences in any other frequency bands.

\section{DISCUSSION}

The aim of the present study was to investigate the nature of the semantic interference effect in the PWI paradigm with regard to the lexical competition account and the response exclusion account by focusing on EEG evidence. We conducted a PWI experiment in which participants named pictures superimposed with semantically related or unrelated written distractor words. Speech onset latencies, error rates, ERPs, and changes in oscillatory activity were investigated. As explained in the introduction, we aimed to avoid any potential re-processing of the stimuli and therefore presented them for a short period of time. In addition, existing EEG studies of PWI had typically considered ERP correlates until about 400/500 ms after picture onset. Similar to Wong et al. (2017), we explored also whether later ERPs as well as oscillatory activity, i.e., those closer to speech onset, were systematically affected by the relationship between targets and distractors. This enabled us to test the prediction of the response exclusion account to find effects close to speech onset caused by the assumed removal of fully planned responses form a response buffer.

In terms of behavioral results we replicated previous findings (e.g., Schriefers et al., 1990). Participants named the pictures more slowly when the distractors were semantically related than when they were unrelated to the targets. In terms of ERPs, only the stimulus-locked analysis showed effects of distractor relatedness. We found several effects of semantic relatedness (i.e., for related relative to unrelated distractors) from around $270 \mathrm{~ms}$ to about $670 \mathrm{~ms}$ post stimulus onset: A widespread negativity around $290 \mathrm{~ms}$, a reduced anterior positivity around $470 \mathrm{~ms}$, a reduced left-posterior positivity around $540 \mathrm{~ms}$, and a reduced anteriorcentral negativity around $660 \mathrm{~ms}$. In terms of oscillatory activity, we found that related distractors led to an increase in midfrontal theta power from about 450 to $540 \mathrm{~ms}$, as well as to decreased high beta power between 40 and $110 \mathrm{~ms}$ and increased beta power between 275 and $340 \mathrm{~ms}$, all post stimulus onset. A responselocked analysis showed that related distractors led to increased high and low beta power at various times before response onset, most importantly a high beta power increase from about -175 to $-155 \mathrm{~ms}$ before speech onset.

In order to decide whether these effects are evidence for the lexical selection account and/or the response exclusion account, one needs estimates of when the accounts predict effects to occur. The lexical competition account assumes effects to occur during lemma retrieval. The response exclusion account is unfortunately not very specific in terms of when exactly the exclusion of the response from the articulatory buffer occurs. But, if fully prepared phonological responses are excluded (Finkbeiner and Caramazza, 2006; Mahon et al., 2007; Janssen et al., 2008; Dhooge and Hartsuiker, 2010, 2012), then effects should be seen from the point when a fully prepared phonological word is available.

Indefrey and Levelt's (2004) and Indefrey's (2011) metaanalyses, while somewhat limited as they are based on Levelt et al. (1999) model of word production, provide timing estimates for the different stages of speech production for a $600 \mathrm{~ms}$ response. According to these estimates, lemma retrieval should start at about $200 \mathrm{~ms}$ after picture presentation, phonological code retrieval at about $275 \mathrm{~ms}$, self-monitoring at around $355 \mathrm{~ms}$ and phonetic encoding at about $455 \mathrm{~ms}$. However, since these estimates are based on a response of $600 \mathrm{~ms}$, they have to be rescaled for our longer response time of $\sim 850 \mathrm{~ms}$. There are two ways of rescaling these estimates, proportional and informed rescaling (Indefrey, 2011; Roelofs and Shitova, 2017). Proportional rescaling assumes that all stages of speech 

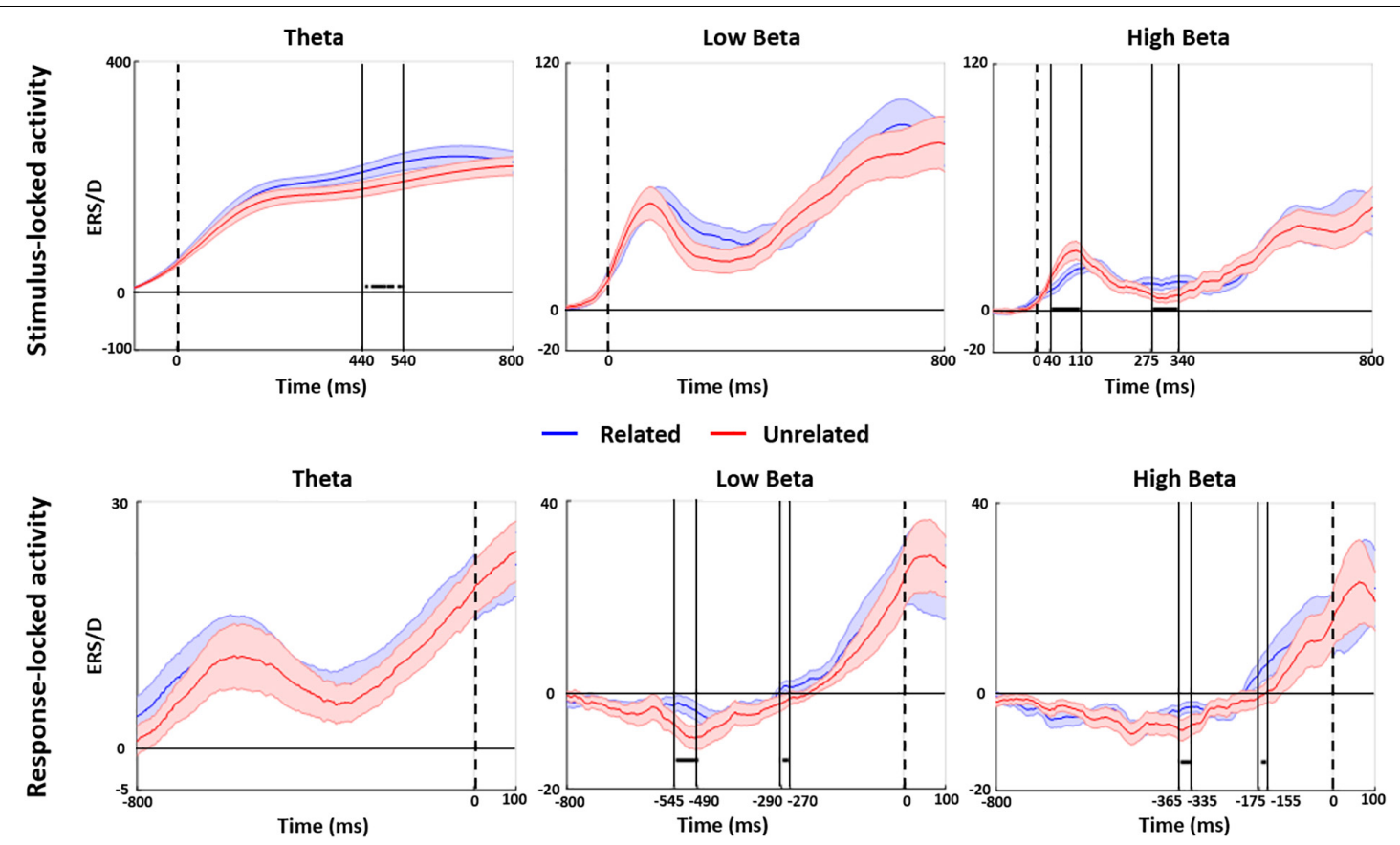

FIGURE 4 | Stimulus and response-locked time-frequency analyses. Point-by-point stimulus-locked (upper panel) and response-locked (lower panel) analyses of related (blue solid line) and unrelated (red solid line) distractors are shown for the FCz electrode with the shaded area of the same color highlighting standard error. Horizontal black thick lines indicate a significant group difference between related vs. unrelated distractor conditions (permutation $t$-test at $p=0.05$ ).

production take longer and therefore all stages contribute to the prolonging of the responses and with equal weight. In contrast, informed rescaling means that only those stages are lengthened that are assumed to take longer in the given paradigm. Since the two approaches lead to very similar estimates for our study, we will focus here on the informed rescaling method. A picture naming study by Bürki (2017), which compared ERPs to pictures with unrelated written distractor words to those without distractors, provides some information for informed rescaling, at least for the first $250 \mathrm{~ms}$ post stimulus onset, that is until the stimuli disappeared in our experiment. Bürki (2017) found differences in ERP amplitudes emerged from about $200 \mathrm{~ms}$ post stimulus presentation. We therefore did not rescale the time window of conceptual preparation (initial $200 \mathrm{~ms}$ ). In addition, we assumed that phonetic encoding would not take longer than in faster responses (final $145 \mathrm{~ms}$ ), given that at this point the speaker has decided which word to produce (but note that rescaling this time window as well does not change the interpretations of our effects described below). Distributing the additional time $(250 \mathrm{~ms})$ that participants in our study took compared to the benchmark time of $600 \mathrm{~ms}$ evenly over the time windows of lemma retrieval and phonological retrieval/syllabification/monitoring would mean that lemma retrieval would last from about 200 to $400 \mathrm{~ms}$, phonological code retrieval and syllabification from about 400 to $705 \mathrm{~ms}$, followed by phonetic encoding. In addition, self-monitoring should start sometime between 500 and $605 \mathrm{~ms}$.

Based on these estimates, the lexical competition account predicts effects in our study around 200 to $400 \mathrm{~ms}$. The response exclusion account predicts effects right before $\sim 700 \mathrm{~ms}$ post stimulus presentation or right before $\sim 150 \mathrm{~ms}$ pre-response. If Dhooge and Hartsuiker $(2010,2012)$ are correct in that the speech monitor is involved in the response exclusion, then effects might also occur from about 500 to $605 \mathrm{~ms}$. But note that this monitor would not work on fully prepared phonological responses as usually assumed in the response exclusion account (Finkbeiner and Caramazza, 2006; Mahon et al., 2007; Janssen et al., 2008; Dhooge and Hartsuiker, 2010, 2012).

We can now compare our findings with these estimates. Our earliest ERP effect $(270-310 \mathrm{~ms})$ falls into the lemma retrieval time window and could therefore be related to increased lexical competition caused by the relatedness of the pictures and distractors (for other studies with the same rationale see Costa et al., 2009; Dell'Acqua et al., 2010). Interestingly, the effect resembles an N400 effect, even though it is much shorter than a typical N400 effect. Therefore, the question arises whether the ERP effect around $290 \mathrm{~ms}$ could also be a pure semantic facilitation effect caused by semantic priming of the related distractor. However, semantic priming and semantic relatedness has been found to lead to reduced negative activation, both in visual word processing and word production studies (e.g., Bentin et al., 1985; Holcomb and Neville, 1990; Chauncey et al., 2009; Blackford et al., 2012). The enhanced negativity of the related distractors compared to the unrelated distractors in our study is not compatible with this suggestion, because the effect is in the opposite direction to the semantic priming effect. It is therefore unlikely that our effect around $290 \mathrm{~ms}$ is a semantic priming instead of a lexical retrieval effect. 
The second ERP effect (440-510 ms) falls into the window of phonological code retrieval and syllabification. The final two ERP effects (520-560 ms and 630-670 ms post stimulus onset) fall into the latter time window as well, but also fit the time window of selfmonitoring (but not of fully prepared phonological responses). These later effects could also be part of a domain-general control mechanism outside language processing, which has been argued to be part of the PWI paradigm (Piai et al., 2013; Janssen et al., 2015). This is especially the case for the two anterior effects around $470 \mathrm{~ms}$ and $660 \mathrm{~ms}$.

For the interpretation of our oscillatory power changes, it is worth discussing what theta and beta power increases have been argued to reflect. As noted in the introduction, an increase in frontal theta power has been related to increased frontal control mechanisms (e.g., Cavanagh and Frank, 2014). The increase in frontal theta power (450-540 ms) falls into the window of phonological code retrieval and syllabification. This increase might therefore reflect the suppression of phonologically encoding the related distractor or the effort of not mixing the phonological codes of picture and distractor.

Beta activity is well known to be decreased in preparation to movements, for instance, limb movements (e.g., Hari and Salmelin, 1997). It has also been found for the preparation of speech production (e.g., Salmelin and Sams, 2002; Saarinen et al., 2006; Gehrig et al., 2012; Jenson et al., 2014). Beta power decrease in these tasks is understood to reflect the preparation of the motor response and the readiness to move. While beta band synchrony has been related to a state of maintenance of posture or status quo (e.g., Engel and Fries, 2010), asynchrony has been related to readiness to move (Gilbertson et al., 2005; Tzagarakis et al., 2010). Response uncertainty in the case of competing potential responses is associated with less reduction of beta power (Tzagarakis et al., 2010). Beta band activity has also been related to cognitive processes. Engel and Fries (2010) have suggested that the active maintenance of a cognitive set and endogenously driven top-down attention processes, for instance in case of ambiguous stimuli, are associated with increase in beta band activity. Similarly, in a review of beta frequency effects in language processing, Weiss and Müller (2012) pointed out that beta power decreases can be related to maintenance of cognitive processes, top-down attention (e.g., attention to more important words) as well as semantic binding during lexical-semantic retrieval processes (for the latter, see, e.g., Luo et al., 2010).

These observations suggest that higher beta power in the related condition versus the unrelated condition in our study might reflect higher response uncertainty, endogenously driven top-down attention processes due to relatedness of the distractor and the maintenance of two active responses, and/or semantic binding during lexical-semantic retrieval processes. Interestingly, beta band effects occurred at various times during response preparation, from at least the timing of lexical retrieval (around $-550 \mathrm{~ms}$ pre-speech onset) right before phonetic encoding (up to $-155 \mathrm{~ms}$ pre speech onset). This suggests that response uncertainty is maintained until phonetic encoding. The earliest beta power difference occurred between 40 and $110 \mathrm{~ms}$ and falls into the time window of visual and semantic processing. This effect seems too early to reflect attention or state maintenance processes, because at this early stage words and pictures are still being recognized. But it cannot fully be ruled out that the processing system is able to adjust attention and maintenance processes with this rudimentary information. Dell'Acqua et al. (2010) suggested for a very early ERP effect that it might reflect feedback processes from the semantics of the picture to the processing of the distractor word. This fits with previous findings that beta decreases have been found for semantic binding and neural binding across domains (here: visual words, pictures and semantics) (see Weiss and Müller, 2012).

How do these results fit with the two accounts of the PWI? In line with the lexical competition account, we found both an ERP and time frequency effect during the time window of lemma retrieval. But given the additional effects, the story seems more complex. A response to the distractor does not seem to be fully suppressed after lemma retrieval. This suggests that the related distractor does not only increase lexical competition during lemma access. Later effects fit with the suggestion by Starreveld and La Heij (1996) that the PWI is related to the selection of the phonological word-form representation and potentially affects self-monitoring processes. While the lexical competition account does not predict our later effects, it needs to be pointed out that 'late' effects of semantic interference can be accounted for in some models of word production that assume lexical competition, like the model by Levelt et al. (1999).

The response exclusion account assumes two effects, i.e., semantic priming of the picture by the related distractor and a late effect when a fully planned response to the distractor needs to be excluded (Finkbeiner and Caramazza, 2006; Mahon et al., 2007; Janssen et al., 2008). As mentioned above, our very early finding of a decrease in high beta power $(40-110 \mathrm{~ms}$ post stimulus onset) might potentially reflect such semantic 'priming' effect. But, as explained above, the ERP effect around $290 \mathrm{~ms}$ is very unlikely a semantic (priming) effect because it is in the opposite direction of a semantic priming effect. In contrast, the increase of high beta band activity between -175 and -155 ms before speech onset and the interpretation of increased beta band power to reflect response uncertainty fits the idea of a response exclusion mechanism of fully formed phonological words for both the picture and the distractor. However, our earlier effects suggest that the response exclusion explanation is not sufficient.

Taken together, our results suggest that semantic distractors affect the processing of the target pictures in more than one way and that both accounts of semantic interference, i.e., lexical competition and response exclusion, seem to capture a part, but not all aspects of the semantic interference effect. This conclusion is in accordance with findings using fMRI (de Zubicaray et al., 2012a,b) and also with the finding that superimposed distractors affect picture naming at various points during response preparation (Bürki, 2017).

Comparing our results with previous findings for semantic distractors in PWI paradigms, our ERP effect around $290 \mathrm{~ms}$ resembles previous results in terms of timing (Dell'Acqua et al., 2010; Aristei et al., 2011; Hoshino and Thierry, 2011; Zhu et al., 2015; Wong et al., 2017). However, the nature of the effect only matches that by Aristei et al. (2011), who report an increased bilateral anterior negativity for related distractors 
relative to unrelated distractors between 200 and $400 \mathrm{~ms}$. In contrast, others found more negative activity for unrelated distractors relative to related distractors in similar time windows (e.g., Dell'Acqua et al., 2010; Hoshino and Thierry, 2011; Zhu et al., 2015; Wong et al., 2017). It is unclear how exactly the differences in the results of the studies arose. Given that our effect was not affected much by our artifact cleaning procedure (Porcaro et al., 2015), speech artifacts do not seem to be responsible for the difference. But procedural differences between the studies might be the cause. For instance, while Dell'Acqua et al. (2010) and Hoshino and Thierry (2011) left the stimuli on the screen, our stimuli disappeared after $250 \mathrm{~ms}$. Aristei et al. (2011) presented distractors auditorily and $-150 \mathrm{~ms}$ before the pictures, meaning early access, but no opportunity for reprocessing of distractors. This is similar to removing distractors early and might explain similarities of their results with ours. However, Aristei et al. (2011) study is also the only study that presented their pictures in semantically homogeneous and heterogeneous blocks. Furthermore, Hoshino and Thierry (2011) study involved bilingual instead of monolingual participants. Such methodological differences might change the way pictures and words interact with each other and/or which words are entering the competition process. Future research will need to investigate whether methodological differences can explain the different ERP results, especially whether keeping the stimuli on the screen until response has an effect on EEG effects.

In terms of oscillatory activity, there are very limited previous results. The short increase in frontal theta power has not been reported before (but see the long-lasting increase in theta power in Shitova et al., 2017, who compared picture of identical with related distractors words). The beta band difference of Piai et al. (2012) for categorically related versus unrelated distractors between 230 and $370 \mathrm{~ms}$ resembles one of our effects, both in terms of nature and timing (275-340 ms). Piai et al. (2012) argued that the effect is in line with the lexical competition account due to its timing. While we agree that this effect is likely related to lexical competition, it is not the only effect in our study, which means it does not tell the whole story.

Overall, our effects seem to be relatively short and small compared to previous findings and compared to our strong behavioral effect. In addition, we found more effects than previous studies. It is possible that this is due to the shortened timing of our stimulus presentation. It is also interesting to see that ERP and time frequency analyses showed effects in partly different time periods, meaning that they seem to reflect different aspects of the underlying processes. Most importantly for the present study, we found an effect close to response onset only in the time frequency domain, not in ERPs. The study of oscillatory activity next to ERPs therefore seems to be a fruitful combination that should be explored more often.

We had previously shown that the SAR-ICA procedure for the removal of speech artifacts (Porcaro et al., 2015) that we applied in this study was very successful in removing speech motor related artifacts from the EEG. It is not certain whether other methods might lead to different results, especially in responselocked analyses. For instance, Ouyang et al. (2016) presented an alternative approach, using residue iteration decomposition.
They argue that their method was superior to an ICA-based method. But even if our method might not have removed all speech motor artifacts from the EEG signal, it is rather unlikely that our effects, including the one close to speech onset, were due to speech motor artifacts. This is because all participants named the same pictures in the related and unrelated conditions and it is unlikely that the procedure would attenuate artifacts better in one than in the other condition.

\section{CONCLUSION}

To conclude, cleaning our data from speech motor artifacts allowed to investigate later processing stages in the PWI paradigm and therefore to address the nature of the semantic distractor effect. In particular, we were able to investigate processes close to speech onset, which are important for the response exclusion account. Our results suggest that the semantic distractor effect is actually a combination of various effects. Lexical competition accounts and the response exclusion account capture different parts, but not all aspects of the behavioral effect. Having said this, our results generally support the traditional conclusion that the PWI paradigm involves competition between categorically related lexical representations and cannot be reduced to later processes such as monitoring processes or a response exclusion mechanism. The results are therefore in line with the idea of lexical selection by competition.

\section{DATA AVAILABILITY}

The datasets generated for this study are available on request to the corresponding author.

\section{AUTHOR CONTRIBUTIONS}

AK designed the study. AK and CP contributed equally to its analysis and write-up. MTM contributed to the analysis of the data.

\section{ACKNOWLEDGMENTS}

We thank Ellen Seiss and Francesca Stregapede, who helped with setting up and conducting the experiment as well as the Experimental Psychology Society (United Kingdom) for financial support. We are also grateful to Antje S. Meyer and Peter Praamstra for their help with the experimental design and their comments on earlier versions of this manuscript. We also thank Linda Wheeldon and reviewers for their comments on earlier versions of this manuscript.

\section{SUPPLEMENTARY MATERIAL}

The Supplementary Material for this article can be found online at: https://www.frontiersin.org/articles/10.3389/fpsyg. 2019.00696/full\#supplementary-material 


\section{REFERENCES}

Abdel Rahman, R., and Melinger, A. (2007). When bees hamper the production of honey: lexical interference from associates in speech production. J. Exp. Psychol. Learn. Mem. Cogn. 33, 604-614. doi: 10.1037/0278-7393.33.3.604

Abdel Rahman, R., and Melinger, A. (2009). Semantic context effects in language production: a swinging lexical network proposal and a review. Lang. Cogn. Process. 24, 713-734. doi: 10.1080/01690960802597250

Acheson, D. J., Hamidi, M., Binder, J. R., and Postle, B. R. (2011). A common neural substrate for language production and verbal working memory. J. Cogn. Neurosci. 23, 1358-1367. doi: 10.1162/jocn.2010.21519

Alario, F. X., Segui, J., and Ferrand, L. (2000). Semantic and associative priming in picture naming. Q. J. Exp. Psychol. Sect. Hum. Exp. Psychol. 53, 741-764. doi: 10.1080/027249800410535

Aristei, S., Melinger, A., and Abdel Rahman, R. (2011). Electrophysiological chronometry of semantic context effects in language production. J. Cogn. Neurosci. 23, 1567-1586. doi: 10.1162/jocn.2010.21474

Barbati, G., Porcaro, C., Hadjipapas, A., Adjamian, P., Pizzella, V., Romani, G. L., et al. (2008). Functional source separation applied to induced visual gamma activity. Hum. Brain Mapp. 29, 131-141. doi: 10.1002/hbm.20375

Barbati, G., Porcaro, C., Zappasodi, F., Rossini, P. M., and Tecchio, F. (2004). Optimization of an independent component analysis approach for artifact identification and removal in magnetoencephalographic signals. Clin. Neurophysiol. 115, 1220-1232. doi: 10.1016/j.clinph.2003.12.015

Bentin, S., McCarthy, G., and Wood, C. C. (1985). Event-related potentials, lexical decision and semantic priming. Electroencephalogr. Clin. Neurophysiol. 60, 343-355. doi: 10.1016/0013-4694(85)90008-2

Blackford, T., Holcomb, P. J., Grainger, J., and Kuperberg, G. R. (2012). A funny thing happened on the way to articulation: N400 attenuation despite behavioral interference in picture naming. Cognition 123, 84-99. doi: 10.1016/j.cognition. 2011.12.007

Bloem, I., and La Heij, W. (2003). Semantic facilitation and semantic interference in word translation: implications for models of lexical access in language production. J. Mem. Lang. 48, 468-488. doi: 10.1016/s0749-596x(02)00503-x

Bullmore, E. T., Suckling, J., Overmeyer, S., Rabe-Hesketh, S., Taylor, E., Brammer, M. J. et al. (1999). Global, voxel, and cluster tests, by theory and permutation, for a difference between two groups of structural MR images of the brain. IEEE Trans. Med. Imaging 18, 32-42. doi: 10.1109/42.750253

Bürki, A. (2017). Electrophysiological characterization of facilitation and interference in the picture-word interference paradigm. Psychophysiology 54, 1370-1392. doi: 10.1111/psyp.12885

Caramazza, A. (1997). How many levels of processing are there in lexical access? Cogn. Neuropsychol. 14, 177-208. doi: 10.1080/026432997381664

Cavanagh, J. F., and Frank, M. J. (2014). Frontal theta as a mechanism for cognitive control. Trends Cogn. Sci. 18, 414-421. doi: 10.1016/j.tics.2014.04.012

Chauncey, K., Holcomb, P. J., and Grainger, J. (2009). Primed picture naming within and across languages: an ERP investigation. Cogn. Affect. Behav. Neurosci. 9, 286-303. doi: 10.3758/cabn.9.3.286

Costa, A., Strijkers, K., Martin, C., and Thierry, G. (2009). The time course of word retrieval revealed by event-related brain potentials during overt speech. Proc. Natl. Acad. Sci. U.S.A. 106, 21442-21446. doi: 10.1073/pnas.0908921106

Cutting, J. C., and Ferreira, V. S. (1999). Semantic and phonological information flow in the production lexicon. J. Exp. Psychol. Learn. Mem. Cogn. 25, 318-344. doi: 10.1037/0278-7393.25.2.318

de Zubicaray, G. I., Hansen, S., and McMahon, K. L. (2012a). Differential processing of thematic and categorical conceptual relations in spoken word production. J. Exp. Psychol. Gen. 142, 131-142. doi: 10.1037/a0028717

de Zubicaray, G. I., Miozzo, M., Johnson, K., Schiller, N. O., and McMahon, K. L. (2012b). Independent distractor frequency and age-of-acquisition effects in picture-word interference: fmri evidence for post-lexical and lexical accounts according to distractor type. J. Cogn. Neurosci. 24, 482-495. doi: 10.1162/jocn_ a_00141

Dell, G. S. (1986). A spreading-activation theory of retrieval in sentence production. Psychol. Rev. 93, 283-321. doi: 10.1037/0033-295X.93.3.283

Dell'Acqua, R., Sessa, P., Peressotti, F., Mulatti, C., Navarrete, E., Grainger, J., et al. (2010). ERP evidence for ultra-fast semantic processing in the picture-word interference paradigm. Front. Psychol. 1:177. doi: 10.3389/fpsyg.2010.00177
Dhooge, E., and Hartsuiker, R. J. (2010). The distractor frequency effect in pictureword interference: evidence for response exclusion. J. Exp. Psychol. Learn. Mem. Cogn. 36, 878-891. doi: 10.1037/a0019128

Dhooge, E., and Hartsuiker, R. J. (2012). Lexical selection and verbal selfmonitoring: effects of lexicality, context, and time pressure in picture-word interference. J. Mem. Lang. 66, 163-176. doi: 10.1016/j.jml.2011.08.004

Engel, A. K., and Fries, P. (2010). Beta-band oscillations - signalling the status quo? Curr. Opin. Neurobiol. 20, 156-165. doi: 10.1016/j.conb.2010.02.015

Finkbeiner, M., and Caramazza, A. (2006). Now you see it, now you don't: on turning semantic interference into facilitation in a Stroop-like task. Cortex 42, 790-796. doi: 10.1016/S0010-9452(08)70419-2

Gehrig, J., Wibral, M., Arnold, C., and Kell, C. A. (2012). Setting up the speech production network: how oscillations contribute to lateralized information routing. Front. Psychol. 3:169. doi: 10.3389/fpsyg.2012.00169

Gilbertson, T., Lalo, E., Doyle, L., Di Lazzaro, V., Cioni, B., Brown, P., et al. (2005). Existing motor state is favored at the expense of new movement during 13-35 $\mathrm{Hz}$ oscillatory synchrony in the human corticospinal system. J. Neurosci. 25, 7771-7779. doi: 10.1523/JNEUROSCI.1762-05.2005

Glaser, W. R., and Düngelhoff, F. J. (1984). The time course of picture word interference. J. Exp. Psychol. Hum. Percept. Perform. 10, 640-654. doi: 10.1037/ 0096-1523.10.5.640

Goldrick, M., and Rapp, B. (2002). A restricted interaction account (RIA) of spoken word production: the best of both worlds. Aphasiology 16, 20-55. doi: 10.1080/ 02687040143000203

Greenham, S. L., and Stelmack, R. M. (2001). Event-related potentials and pictureword naming: effects of attention and semantic relation for children and adults. Dev. Neuropsychol. 20, 619-638. doi: 10.1207/S15326942DN2003_5

Groppe, D. M., Urbach, T. P., and Kutas, M. (2011). Mass univariate analysis of event-related brain potentials/fields I: a critical tutorial review. Psychophysiology 48, 1711-1725. doi: 10.1111/j.1469-8986.2011.01273.x

Hari, R., and Salmelin, R. (1997). Human cortical oscillations: a neuromagnetic view through the skull. Trends Neurosci. 20, 44-49. doi: 10.1016/S01662236(96)10065-5

Hirschfeld, G., Jansma, B., Bölte, J., and Zwitserlood, P. (2008). Interference and facilitation in overt speech production investigated with event-related potentials. Neuroreport 19, 1227-1230. doi: 10.1097/WNR.0b013e328309ecd1

Holcomb, P. J., and Neville, H. J. (1990). Auditory and visual semantic priming in lexical decision - a comparison using event-related brain potentials. Lang. Cogn. Process. 5, 281-312. doi: 10.1080/01690969008407065

Hoshino, N., and Thierry, G. (2011). Language selection in bilingual word production: electrophysiological evidence for cross-language competition. Brain Res. 1371, 100-109. doi: 10.1016/j.brainres.2010.11.053

Indefrey, P. (2011). The spatial and temporal signatures of word production components: a critical update. Front. Psychol. 2:255. doi: 10.3389/fpsyg.2011. 00255

Indefrey, P., and Levelt, W. J. M. (2004). The spatial and temporal signatures of word production components. Cognition 92, 101-144. doi: 10.1016/j.cognition. 2002.06.001

Janssen, N., Hernández-Cabrera, J. A., van der Meij, M., and Barber, H. A. (2015). Tracking the time course of competition during word production: evidence for a post-retrieval mechanism of conflict resolution. Cereb. Cortex 25, 2960-2969. doi: 10.1093/cercor/bhu092

Janssen, N., Schirm, W., Mahon, B. Z., and Caramazza, A. (2008). Semantic interference in a delayed naming task: evidence for the response exclusion hypothesis. J. Exp. Psychol. Learn. Mem. Cogn. 34, 249-256. doi: 10.1037/02787393.34.1.249

Jenson, D., Bowers, A. L., Harkrider, A. W., Thornton, D., Cuellar, M., Saltuklaroglu, T., et al. (2014). Temporal dynamics of sensorimotor integration in speech perception and production: independent component analysis of EEG data. Front. Psychol. 5:656. doi: 10.3389/fpsyg.2014. 00656

La Heij, W., and Van den Hof, E. (1995). Picture word interference increases with target set size. Psychol. Res. Psychol. Forsch. 58, 119-133. doi: 10.1007/ BF00571100

Levelt, W. J. M., Roelofs, A., and Meyer, A. S. (1999). A theory of lexical access in speech production. Behav. Brain Sci. 22, 1-75. doi: 10.1017/ S0140525X99001776 
Luo, Y., Zhang, Y., Feng, X., and Zhou, X. (2010). Electroencephalogram oscillations differentiate semantic and prosodic processes during sentence reading. Neuroscience 169, 654-664. doi: 10.1016/j.neuroscience.2010.05.032

Lupker, S. J. (1979). Semantic nature of response competition in the picture-word interference task. Mem. Cogn. 7, 485-495. doi: 10.3758/CABN.9.3.260

Mahon, B. Z., Costa, A., Peterson, R., Vargas, K. A., and Caramazza, A. (2007). Lexical selection is not by competition: a reinterpretation of semantic interference and facilitation effects in the picture-word interference paradigm. J. Exp. Psychol. Learn. Mem. Cogn. 33, 503-535. doi: 10.1037/02787393.33.3.503

Makeig, S., Debener, S., Onton, J., and Delorme, A. (2004). Mining event-related brain dynamics. Trends Cogn. Sci. 8, 204-210. doi: 10.1016/j.tics.2004.03.008

Medaglia, M. T., Tecchio, F., Seri, S., Di Lorenzo, G., Rossini, P. M., Porcaro, C., et al. (2009). Contradition in universal and particular resasoning. Hum. Brain Mapp. 30, 4187-4197. doi: 10.1002/hbm.20838

Miozzo, M., and Caramazza, A. (2003). When more is less: a counterintuitive effect of distractor frequency in the picture-word interference paradigm. J. Exp. Psychol. Gen. 132, 228-252. doi: 10.1037/0096-3445.132.2.228

Oldfield, R. C. (1971). The assessment and analysis of handedness: the edinburgh inventory. Neuropsychologia 9, 97-113. doi: 10.1016/0028-3932(71)90067-4

Oostenveld, R., and Praamstra, P. (2001). The five percent electrode system for high-resolution EEG and ERP measurements. Clin. Neurophysiol. 112, 713-719. doi: 10.1016/S1388-2457(00)00527-7

Ouyang, G., Sommer, W., Zhou, C., Aristei, S., Pinkpank, T., Abdel Rahman, R., et al. (2016). Articulation artifacts during overt language production in eventrelated brain potentials: description and correction. Brain Topogr. 29, 791-813. doi: 10.1007/s10548-016-0515-1

Piai, V., Roelofs, A., Acheson, D. J., and Takashima, A. (2013). Attention for speaking: domain-general control from the anterior cingulate cortex in spoken word production. Front. Hum. Neurosci. 7:832. doi: 10.3389/fnhum.2013.00832

Piai, V., Roelofs, A., Jensen, O., Schoffelen, J.-M., and Bonnefond, M. (2014). Distinct patterns of brain activity characterise lexical activation and competition in spoken word production. PLoS One 9:e88674. doi: 10.1371/ journal.pone.0088674

Piai, V., Roelofs, A., and van der Meij, R. (2012). Event-related potentials and oscillatory brain responses associated with semantic and Stroop-like interference effects in overt naming. Brain Res. 1450, 87-101. doi: 10.1016/j. brainres.2012.02.050

Porcaro, C., Balsters, J. H., Mantini, D., Robertson, I. H., and Wenderoth, N. (2019). $\mathrm{P} 3 \mathrm{~b}$ amplitude as a signature of cognitive decline in the older population: an EEG study enhanced by functional source separation. NeuroImage 184, 535-546. doi: 10.1016/j.neuroimage.2018.09.057

Porcaro, C., Coppola, M., Di Lorenzo, G., Zappasodi, F., SIracusano, A., Pierelli, F., et al. (2009). Hand somatosensory subcortical and scortical sources assessed by functional source separation: an EEG study. Hum. Brain Mapp. 30, 660-674. doi: $10.1002 / \mathrm{hbm} .20533$

Porcaro, C., Di Lorenzo, G., Seri, S., Pierelli, F., Tecchio, F., Coppola, G., et al. (2017). Impaired brainstem and thalamic high-frequency oscillatory EEG activity in migraine between attacks. Cephalalgia 37, 915-926. doi: 10.1177/ 0333102416657146

Porcaro, C., Medaglia, M. T., and Krott, A. (2015). Removing speech artifacts from electroencephalographic recordings during overt picture naming. NeuroImage 105, 171-180. doi: 10.1016/j.neuroimage.2014.10.049

Porcaro, C., Ostwald, D., Hadjipapas, A., Barnes, G. R., and Bagshaw, A. P. (2011). The relationship between the visual evoked potential and the gamma band investigated by blind and semi-blind methods. NeuroImage 56, 1059-1071. doi: 10.1016/j.neuroimage.2011.03.008

Porcaro, C., Zappasodi, F., Barbati, G., Salustri, C., Pizzella, V., Rossini, P. M., et al. (2006). Fetal auditory respones to external sounds and mother's heart beat: detection improved by independent component analysis. Brain Res. 1101, 51-58. doi: 10.1016/j.brainres.2006.04.134

Roelofs, A. (1992). A spreading-activation theory of lemma retrieval in speaking. Cognition 42, 107-142. doi: 10.1016/0010-0277(92)90041-F

Roelofs, A., Piai, V., and Schriefers, H. (2011). Selective attention and distractor frequency in naming performance: comment on dhooge and hartsuiker (2010). J. Exp. Psychol. Learn. Mem. Cogn. 37, 1032-1038. doi: 10.1037/a0023328
Roelofs, A., and Shitova, N. (2017). Importance of response time in assessing the cerebral dynamics of spoken word production: comment on Munding et al. (2016). Lang. Cogn. Neurosci. 32, 1064-1067. doi: 10.1080/23273798.2016. 1274415

Rose, S. B., Aristei, S., Melinger, A., and Abdel Rahman, R. (2018). The closer they are, the more they interfere: semantic similarity of word distractors increases competition in language production. J. Exp. Psychol. Learn. Mem. Cogn. doi: 10.1037/xlm0000592 [Epub ahead of print].

Saarinen, T., Laaksonen, H., Parviainen, T., and Salmelin, R. (2006). Motor cortex dynamics in visuomotor production of speech and non-speech mouth movements. Cereb. Cortex 16, 212-222. doi: 10.1093/cercor/bhi099

Salmelin, R., and Sams, M. (2002). Motor cortex involvement during verbal versus non-verbal lip and tongue movements. Hum. Brain Mapp. 16, 81-91. doi: 10.1002/hbm.10031

Schriefers, H., Meyer, A. S., and Levelt, W. J. M. (1990). Exploring the time course of lexical access in language production - picture-word interference studies. J. Mem. Lang. 29, 86-102. doi: 10.1016/0749-596X(90)90011-N

Shitova, N., Roelofs, A., Schriefers, H., Bastiaansen, M., and Schoffelen, J. M. (2016). Using brain potentials to functionally localise stroop-like effects in colour and picture naming: perceptual encoding versus word planning. PLoS One 11:16. doi: 10.1371/journal.pone.0161052

Shitova, N., Roelofs, A., Schriefers, H., Bastiaansen, M., and Schoffelen, J. M. (2017). Control adjustments in speaking: electrophysiology of the gratton effect in picture naming. Cortex 92, 289-303. doi: 10.1016/j.cortex.2017. 04.017

Snodgrass, J. G., and Vanderwart, M. (1980). Standardized set of 260 pictures - norms for name agreement, image agreement, familiarity, and visual complexity. J. Exp. Psychol. Hum. Learn. Mem. 6, 174-215. doi: 10.1037/02787393.6.2.174

Starreveld, P. A., and La Heij, W. (1996). Time-course analysis of semantic and orthographic context effects in picture naming. J. Exp. Psychol. Learn. Mem. Cogn. 22, 896-918. doi: 10.1037/0278-7393.22. 4.896

Tzagarakis, C., Ince, N. F., Leuthold, A. C., and Pellizzer, G. (2010). Betaband activity during motor planning reflects response uncertainty. J. Neurosci. 30, 11270-11277. doi: 10.1523/JNEUROSCI.6026-09. 2010

Weiss, S., and Müller, H. (2012). Too many betas do not spoil the broth: the role of beta brain oscillations in language processing. Front. Psychol. 3:201. doi: 10.3389/fpsyg.2012.00201

Wilson, S. M., Isenberg, A. L., and Hickok, G. (2009). Neural correlates of word production stages delineated by parametric modulation of psycholinguistic variables. Hum. Brain Mapp. 30, 3596-3608. doi: 10.1002/hbm.20782

Wong, A. W. K., Chiu, H. C., Wang, J., Cao, J. L., Wong, S. S., and Chen, H. C. (2017). An early locus of associative and categorical context effects in speech production: evidence from an ERP study using the picture-word interference paradigm. Lang. Cogn. Neurosci. 32, 1305-1319. doi: 10.1080/23273798.2017. 1355060

Zhu, X., Damian, M. F., and Zhang, Q. (2015). Seriality of semantic and phonological processes during overt speech in Mandarin as revealed by event-related brain potentials. Brain Lang. 144, 16-25. doi: 10.1016/j.bandl.2015.03.007

Zwitserlood, P., Bolte, J., and Dohmes, P. (2000). Morphological effects on speech production: evidence from picture naming. Lang. Cogn. Process. 15, 563-591. doi: 10.1080/01690960050119706

Conflict of Interest Statement: The authors declare that the research was conducted in the absence of any commercial or financial relationships that could be construed as a potential conflict of interest.

Copyright (c) 2019 Krott, Medaglia and Porcaro. This is an open-access article distributed under the terms of the Creative Commons Attribution License (CC BY). The use, distribution or reproduction in other forums is permitted, provided the original author(s) and the copyright owner(s) are credited and that the original publication in this journal is cited, in accordance with accepted academic practice. No use, distribution or reproduction is permitted which does not comply with these terms. 
Krott et al.

Semantic Distractors During Picture Naming

APPENDIX A: EXPERIMENTAL MATERIAL

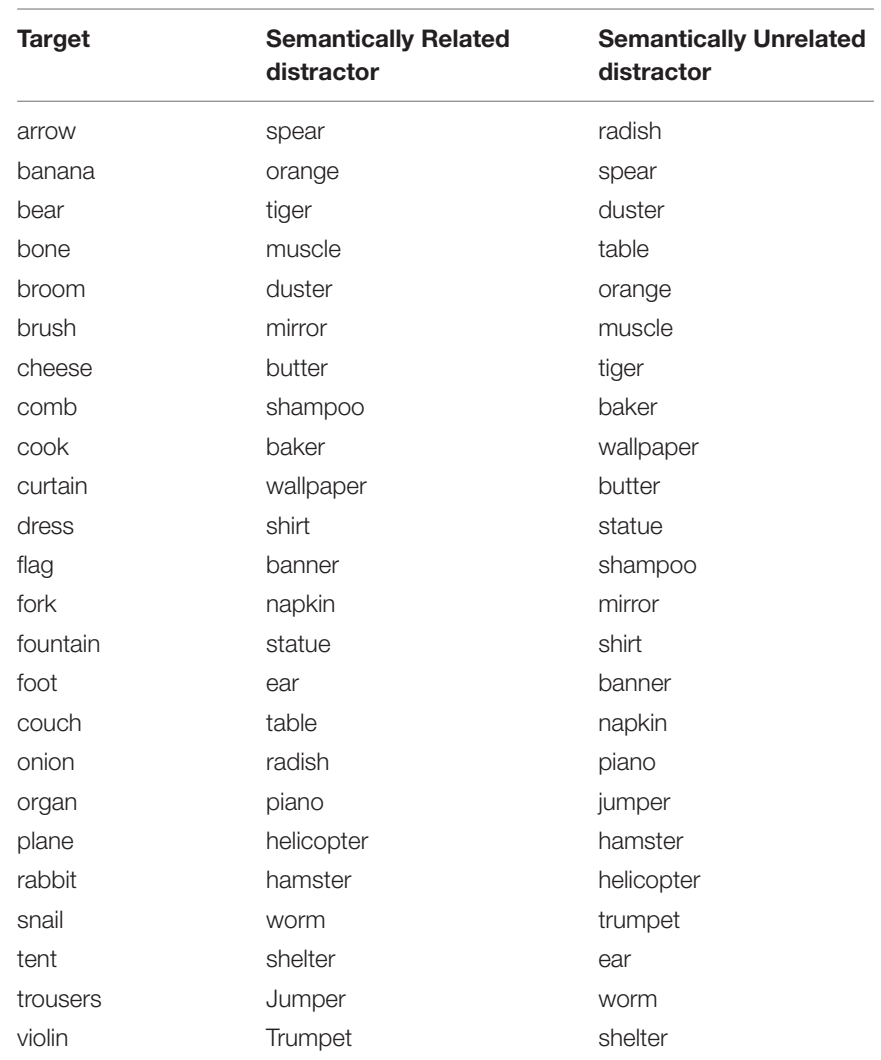

Frontiers in Psychology | www. frontiersin.org

13

March 2019 | Volume 10 | Article 696 